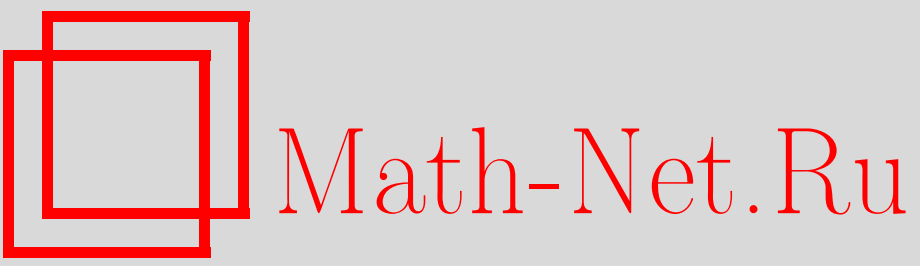

М. А. Урусов, Об оптимальном прогнозе момента достижения максимума броуновским движением, УМН, 2002, том 57, выпуск 1, 165-166

DOI: https://doi.org/10.4213/rm487

Использование Общероссийского математического портала Math-Net.Ru подразумевает, что вы прочитали и согласны с пользовательским соглашением

http://www.mathnet.ru/rus/agreement

Параметры загрузки:

IP: 52.6 .47 .48

26 апреля 2023 г., 09:54:15 


\title{
ОБ ОПТИМАЛЬНОМ ПРОГНОЗЕ МОМЕНТА ДОСТИЖЕНИЯ МАКСИМУМА БРОУНОВСКИМ ДВИЖЕНИЕМ
}

\author{
M. А. УРусОв
}

1. Рассмотрим вероятностное пространство $(\Omega, \mathscr{F}, \mathrm{P})$ и стандартное броуновское движение $B=\left(B_{t}(\omega)\right)_{0 \leqslant t \leqslant 1}$ на нем. Пусть $\left(\mathscr{F}_{t}\right)_{0 \leqslant t \leqslant 1}-$ фильтрация, порожденная $B$ и пополненная множествами Р-меры 0 . Символом $\theta=\theta(\omega)$ обозначим момент, когда $B$ достигнет своего максимального значения: $B_{\theta}=\max _{0 \leqslant t \leqslant 1} B_{t}$. Такой момент $\theta$ определяется Р-п.н. однозначно. $\left(\mathscr{F}_{t}\right)$-моменты остановки будем обозначать через $\tau, \widetilde{\tau}, \tau_{*}, \ldots$ Всюду далее мы рассматриваем в качестве временного интервала отрезок $[0,1]$, поэтому все рассматриваемые моменты остановки $\tau$ предполагаются удовлетворяющими условию $0 \leqslant \tau \leqslant 1$.

В данной работе решены следующие задачи.

ЗАДАчА 1 (байесовская задача). Для произвольного $\lambda>0$ найти оптимальный момент остановки $\tau_{*}(\lambda)$, на котором достигается инфимум

$$
R(\lambda)=\inf _{\tau} \mathrm{E}\left[(1-\tau) I_{\{\tau<\theta\}}+\lambda(\tau-\theta)^{+}\right] .
$$

ЗАДАчА 2 (условно экстремальная задача). Для произвольного $0 \leqslant \alpha \leqslant 1$ найти оптимальныц иомент остановки $\tau(\alpha)$, на котором достигается инфимум

$$
r(\alpha)=\inf _{\tau \in \mathfrak{N}(\alpha)} \mathrm{E}(\tau-\theta)^{+}
$$

в классе $\mathfrak{N}(\alpha)=\left\{\tau: \mathrm{E}\left[(1-\tau) I_{\{\tau<\theta\}}\right] \leqslant \alpha\right\}$.

2. Для формулировки результатов обозначим через $S=\left(S_{t}\right)_{0 \leqslant t \leqslant 1}$ процесс максимума броуновского движения: $S_{t}=\max _{0 \leqslant s \leqslant t} B_{s}$. Пусть $\varphi(x)$ и $\Phi(x)$ - соответственно плотность и функция распределения нормальной случайной величины с нулевым средним и единичной дисперсией.

ТеОрема 1. В байесовской задаче существ ует единственный оптимальный момент остановки $\tau_{*}(\lambda)$, и он дается формулой

$$
\tau_{*}(\lambda)=\inf \left\{t \geqslant 0: S_{t}-B_{t} \geqslant z_{*}(\lambda) \sqrt{1-t}\right\},
$$

где $z_{*}(\lambda)$ - единственный положительный корень уравнения

$$
2(\lambda+1) z[\Phi(z)-1 / 2]-\left[(\lambda+1) z^{2}+1\right] \varphi(z)-z=0 .
$$

ЗАмечАниЕ. Можно показать, что функция $z_{*}(\lambda), \lambda \in(0,+\infty)$, строго убывает, непрерьвна, $z_{*}(\lambda) \underset{\lambda \downarrow 0}{\longrightarrow}+\infty, z_{*}(\lambda) \underset{\lambda \rightarrow+\infty}{\longrightarrow} 0$.

Отсюда и из вида оптимального момента остановки следует, что

$$
\begin{aligned}
& \lambda_{1}<\lambda_{2} \Rightarrow z_{*}\left(\lambda_{1}\right)>z_{*}\left(\lambda_{2}\right) \Rightarrow \tau_{*}\left(\lambda_{1}\right)>\tau_{*}\left(\lambda_{2}\right) ; \\
& \lambda \uparrow+\infty \Rightarrow z_{*}(\lambda) \downarrow 0 \Rightarrow \tau_{*}(\lambda) \downarrow 0 ; \\
& \lambda \downarrow 0 \Rightarrow z_{*}(\lambda) \uparrow+\infty \Rightarrow \tau_{*}(\lambda) \uparrow 1 .
\end{aligned}
$$

Это вполне согласуется с интуицией: если $\lambda$ велико, то штрафф за опоздание большой, поэтому надо раньше останавливаться; и все наоборот, если $\lambda$ мало.

ТЕОРема 2. В условно экстремальной задаче существ ует единственный оптимальный момент остановки $\tau(\alpha)$, и он дается следующими формулами:

$$
\text { для } \quad 0<\alpha<1 \quad \tau(\alpha)=\tau_{*}(\lambda(\alpha)),
$$

где $\lambda(\alpha)$ - единственный положительный корень уравнения

$$
\begin{array}{ccc}
2 \frac{1-\Phi\left(z_{*}(\lambda)\right)}{1+\left(z_{*}(\lambda)\right)^{2}}=\alpha ; \\
\partial \Omega Я \quad \alpha=0 & \tau(0)=1 ; \\
\partial \Omega Я \quad & \alpha=1 & \tau(1)=0 .
\end{array}
$$


3. Сформулированные задачи возникают в финансовой математике. Для анализа финансов естественна следующая постановка: как на основании последовательных наблюдений цен акций на конечном отрезке времени выбрать момент, которьй в некотором смысле наиболее близок к моменту достижения ценами акций максимального значения. Байесовская задача - частный случай такой постановки, где в качестве цен акций рассматривается броуновское движение и (1) определяет смысл, в котором измеряется близость между $\tau$ и $\theta$. Именно, задача состоит в том, чтобы минимизировать среднее значение штраффа, где штраф̆ за раннюю остановку $(\tau<\theta)$ составляет $(1-\tau) I_{\{\tau<\theta\}}$, а за опоздание $(\tau>\theta)-\lambda(\tau-\theta)^{+}$. Условно экстремальная задача - тоже частный случай рассмотренной выше постановки. В ней надо минимизировать штрафф за опоздание при условии, что нельзя "слишком рано" останавливаться (штраф̆ за раннюю остановку не должен превосходить $\alpha$ ).

Отсюда видно, что было бы интересно найти оптималњный момент остановки, на котором достигается inf $\tau \mathrm{E}|\tau-\theta|$. Эта задача мажорируется байесовской в том смысле, что

$$
\inf _{\tau} \mathrm{E}|\tau-\theta| \leqslant R(1)
$$

Первый результат в этом направлении установлен в [1], где решена задача

$$
\text { "inf } \mathrm{E}\left(B_{\theta}-B_{\tau}\right)^{2} \quad\left(=\inf _{\tau} \mathrm{E}\left(\max _{0 \leqslant t \leqslant 1} B_{t}-B_{\tau}\right)^{2}\right) " .
$$

Интересно отметить, что оптимальньй момент остановки в ней тоже имеет вид (2).

4. Рассмотрим байесовскую и условно экстремальную задачи для процесса $X_{t}=f\left(B_{t}\right)$ вместо броуновского движения $B_{t}$, где $f$ - детерминированная строго возрастающая функция.

Момент, когда $X_{t}$ достигает своего максимального значения на отрезке $[0,1]$, совпадает с $\theta$ - соответствующим моментом для $B_{t}$. Фильтрации, порожденные $X$ и $B$, совпадают. Следовательно, $\tau$ - момент остановки относительно $X$, если и только если $\tau$ - момент остановки относителњно $B$.

Отсюда ясно, как переформулировать теоремы 1 и 2 , чтобы получить решения соответствующих задач.

В частности, это замечание показьвает, что установлены аналогичные результаты для процесса $e^{B_{t}}$ вместо $B_{t}$. С финансовой точки зрения они более привлекательны, поскольку процесс $e^{B_{t}}$ болшше похож на цены акций, чем броуновское движение, так как он строго положителен.

\section{СПИСОК ЛИТЕРАТУРЫ}

[1] S. E. Graversen, G. Peskir, A. N. Shiryaev // Теория вероятн. и ее примен. 2000. T. 45. № 1. C. 125-136.

Московский государственньй

Принято редколлегией университет им. М.В. Ломоносова 26.12 .2001

E-mail: urusov@mech.math.su 